\title{
Quantitative Bone Single Photon Emission Computed Tomography/Computed Tomography for Evaluating Response to Bisphosphonate Treatment in Patients with Paget's Disease of Bone
}

\author{
Kazuhiro Kitajima ${ }^{a}$ Hiroyuki Futani $^{b}$ Tatsuya Tsuchitanic ${ }^{c}$ Yoshiyuki Takahashic ${ }^{c}$ \\ Koichiro Yamakado a \\ aDepartment of Radiology, Hyogo College of Medicine, Nishinomiya, Japan; \\ ${ }^{b}$ Department of Orthopedic Surgery, Hyogo College of Medicine, Nishinomiya, Japan; \\ 'Department of Radiological Technology, Hyogo College of Medicine College Hospital, \\ Nishinomiya, Japan
}

Keywords
Paget's disease of bone · Bisphosphonate $\cdot$ Treatment response $\cdot$ Bone scintigraphy
Quantitative SPECT/CT

\section{Abstract}

We here report on 2 cases of monostotic Paget's disease of bone, one in the ilium and the other in the skull, including quantitative values obtained with bone single photon emission computed tomography/computed tomography (SPECT/CT), which were useful to evaluate the response to bisphosphonate treatment. The quantitative parameters determined with those findings were decreased, with the maximum standardized uptake value (SUV), peak SUV, mean SUV, metabolic bone volume, and total bone uptake in case 1 and case 2 reduced by 48.8 and $60.3 \%, 46.6$ and $58.8 \%, 24.3$ and $60.5 \%, 87.0$ and $11.8 \%$, and 90.2 and $55.8 \%$, respectively, while TRACP-5B and alkaline phosphatase (ALP) were also reduced by 39.5 and $88.6 \%$ and by 53.7 and $78.1 \%$, respectively. Quantitative SPECT/CT parameter decreases were correlated with TRACP-5B and ALP, indicating the usefulness of this modality to examine treatment response.

\section{Introduction}

Paget's disease of bone (PDB) is a benign disease characterized by accelerated turnover, that is, bone resorption and formation [1]. Modern treatment methods rely on bisphosphonates, which are powerful blockers of bone remodeling [1]. Radionuclide bone scintigraphy 
with technetium-99m ( ${ }^{99 \mathrm{~m}} \mathrm{Tc}$ ) can evaluate osteoblastic activity, and is a widely used valuable technique for assessments of disease extent and treatment response to bisphosphonates in patients with PDB [1].

However, abnormal uptake in bone observed with planar bone scintigraphy cannot be easily and reliably quantified, and semiquantitative visual methods are not adequately sensitive to measure the actual response, even with images acquired using single photon emission computed tomography (SPECT). On the other hand, recent advances have resulted in integration of computed tomography (CT) for attenuation correction together with a sophisticated reconstruction technique, which has enabled quantitative measurements with SPECT/CT suitable for derivation of the standardized uptake value (SUV) [2-4]. It is considered that quantitative SPECT/CT may soon have an enormous effect as an imaging biomarker in the field of modern nuclear medicine and for clinical practice.

Here, we report on 2 cases of PDB in which quantitative values were determined with bone SPECT/CT measurements and found useful to evaluate treatment response to bisphosphonates.

\section{Case Report}

\section{Case 1}

A 62-year-old female came to our hospital with left hip pain, and the prior medical history included chronic renal failure, hypertension, hyperlipidemia, and hyperuricemia. CT imaging showed irregular cortical thickening and swelling of the left ilium with osteosclerosis and ground-glass opacity (Fig. 1a), while magnetic resonance imaging revealed heterogeneous hypointensity of the lesion in T1- and T2-weighted images (Fig. 1b-d). Additionally, ${ }^{99 \mathrm{~m}} \mathrm{Tc}$ hydroxymethylene diphosphonate (HMDP) bone SPECT/CT showed focal and intense uptake by the bony lesion (Fig. 1e). Serum TRACP-5B and total alkaline phosphatase (ALP) before beginning bisphosphonate treatment were $780 \mathrm{mU} / \mathrm{dL}$ (reference range: 120-420) and 415 U/L (reference range: 44-147), respectively. The patient was diagnosed with PDB in the left ilium and began alendronate sodium hydrate therapy ( $35 \mathrm{mg}$, once every 2 weeks). After 1 year of treatment, TRACP-5B and ALP were decreased to $472 \mathrm{mU} / \mathrm{dL}$ (39.5\% reduction) and $192 \mathrm{U} / \mathrm{L}$ (53.7\% reduction), respectively, along with significant clinical improvement. A second ${ }^{99 \mathrm{~m} T c-H M D P}$ bone SPECT/CT examination revealed a decrease in focal uptake in the left ilium (Fig. 1e).

SPECT/CT scanning was performed using an integrated SPECT/CT system (Discovery NM/CT 670; GE Healthcare) equipped with a low-energy high-resolution collimator $3 \mathrm{~h}$ after intravenous injection of $440 \mathrm{MBq}$ of ${ }^{99 \mathrm{~m}} \mathrm{Tc}$-HMDP. The obtained data were analyzed using a commercially available software package (GI-BONE; Aze Co., Ltd., Tokyo, Japan), which presents various SUVs, including $\mathrm{SUV}_{\text {max }}, \mathrm{SUV}_{\text {peak, }}$ and $\mathrm{SUV}_{\text {mean }}$, as well as metabolic bone volume (MBV) and total bone uptake (TBU) values. MBV was defined as tumor volume with

Fig. 1. Case 1,62-year-old female with Paget's disease of bone in the left ilium. a Pre-treatment unenhanced computed tomography (CT) image (bone window) showing irregular cortical thickening and swelling of the left ilium with osteosclerosis and ground-glass opacity. b Pre-treatment axial T1-weighted magnetic resonance (MR) image showing heterogeneous hypointensity of the lesion. c Pre-treatment axial T2-weigthed MR image showing heterogeneous hypointensity of the lesion. $\mathbf{d}$ Pre-treatment coronal T1-weighted MR image showing heterogeneous hypointensity of the lesion. e Pre-treatment whole-body bone scintigraphy showing strong accumulation of ${ }^{99 \mathrm{~m}} \mathrm{Tc}$-hydroxymethylene diphosphonate ( $\left.{ }^{99 \mathrm{~m}} \mathrm{Tc}-\mathrm{HMDP}\right)$ in the left pelvic bone, and bone single photon emission computed tomography/CT (SPECT/CT) image showing strong focal ${ }^{99 \mathrm{~m}} \mathrm{Tc}-\mathrm{HM}$ DP uptake in the left ilium. Post-treatment whole-body bone scintigraphy shows a decrease in focal ${ }^{99 \mathrm{~m} T c-}$ HMDP uptake in the left pelvic bone, and bone SPECT/CT shows a decrease in focal ${ }^{99 \mathrm{~m}} \mathrm{Tc}$-HMDP uptake in the left ilium.

(For figure see next page.)

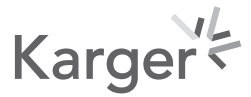




\section{Case Reports in Oncology}

\begin{tabular}{|c|c|}
\hline Case Rep Oncol 2020;13:82 & \\
\hline DOl: 10.1159/000507663 & $\begin{array}{l}\text { (9) } 2020 \text { The Author(s). Published by S. Karger AG, Basel } \\
\text { www.karger.com/cro }\end{array}$ \\
\hline
\end{tabular}

Kitajima et al.: Quantitative SPECT/CT for Response to Bisphosphonate in PDB
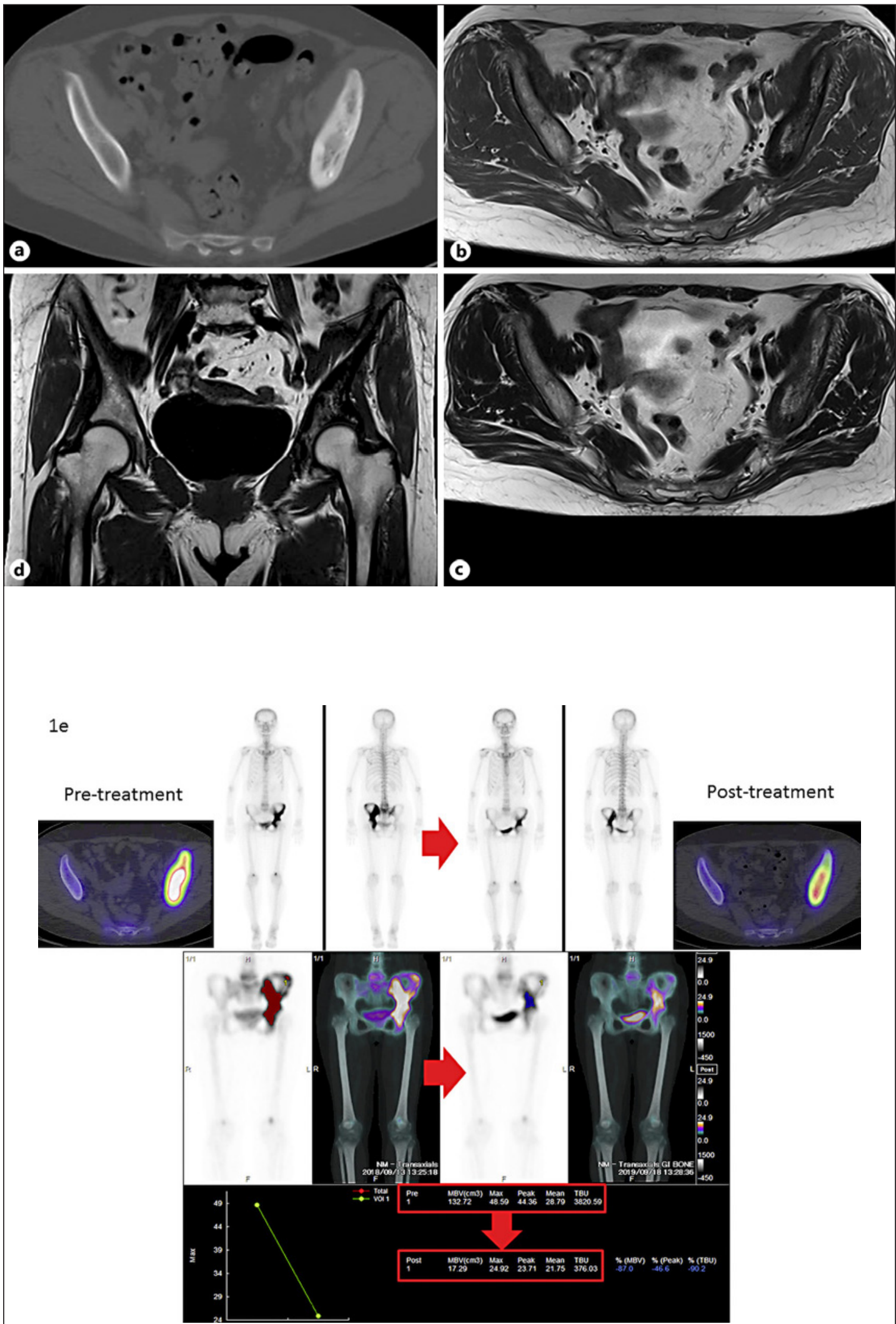

Karger's 


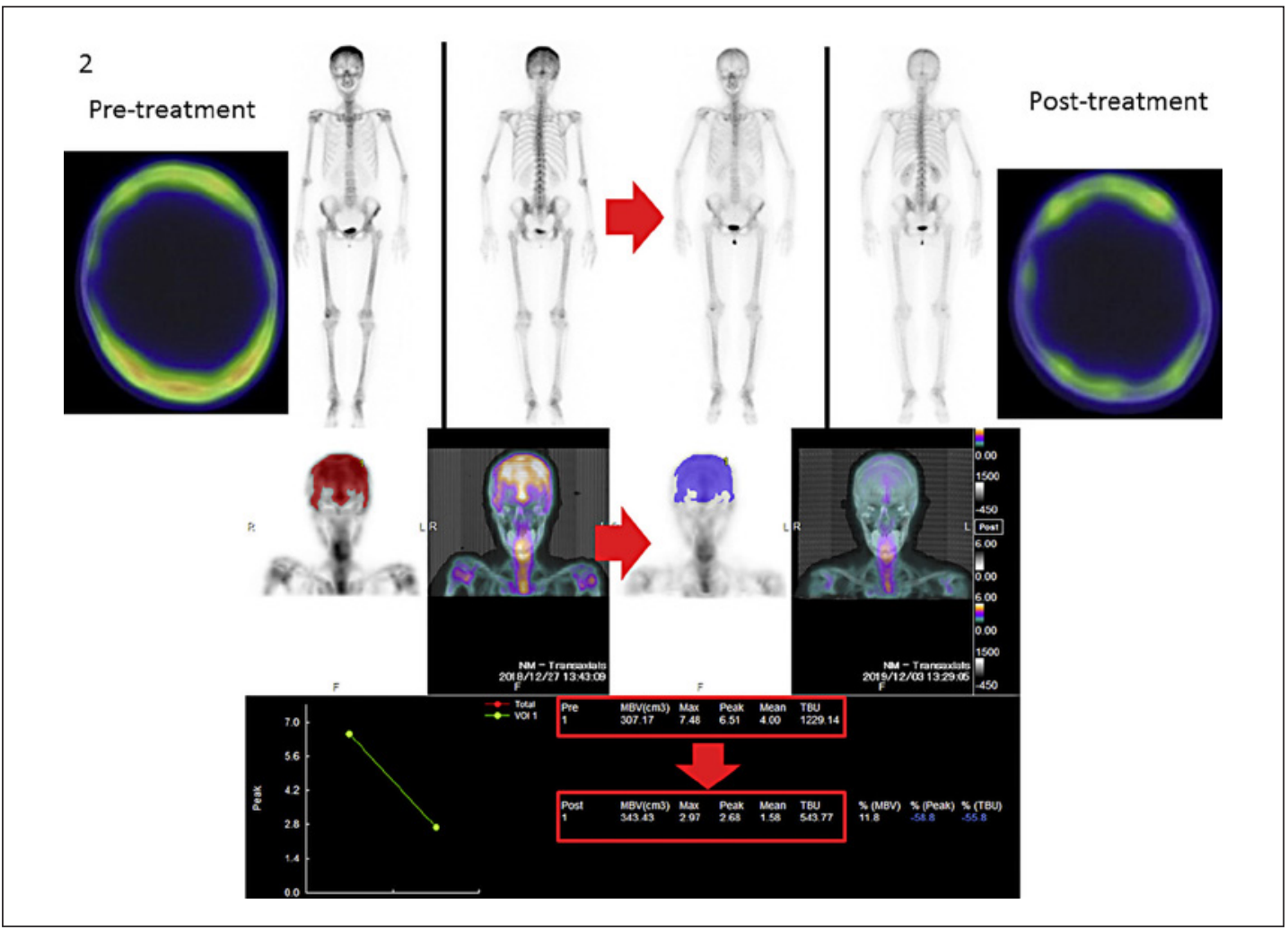

Fig. 2. Case 2, 55-year-old female with Paget's disease of bone in the skull. Pre-treatment whole-body bone scintigraphy shows strong accumulation of ${ }^{99 \mathrm{~m}} \mathrm{Tc}$-hydroxymethylene diphosphonate ( ${ }^{99 \mathrm{~m}} \mathrm{Tc}$-HMDP) in the skull, and the bone single photon emission computed tomography/computed tomography (SPECT/CT) image shows focal ${ }^{99 \mathrm{~m}} \mathrm{Tc}$-HMDP uptake in the skull. Post-treatment whole-body bone scintigraphy shows a decrease in focal ${ }^{99 \mathrm{~m}} \mathrm{Tc}-\mathrm{HMDP}$ uptake in the skull, and the bone SPECT/CT image shows a decrease in ${ }^{99 \mathrm{~m}} \mathrm{Tc}-$ HMDP uptake in the skull.

uptake, while TBU was calculated as $\mathrm{SUV}_{\text {mean }} \times \mathrm{MBV}$. Prior to treatment, $\mathrm{SUV}_{\text {max }}, \mathrm{SUV}_{\text {peak }}$, and $\mathrm{SUV}_{\text {mean }}$ were 48.6, 44.4, and 28.8, respectively, while the values for MBV and TBU were 132.7 $\mathrm{mL}$ and 3,820.6 g, respectively. Following bisphosphonate treatment (alendronate sodium hydrate), those parameters were decreased to $24.9,23.7,21.8,17.3 \mathrm{~mL}$, and $376.0 \mathrm{~g}$, respectively, representing a reduction of $48.8,46.6,24.3,87.0$, and $90.2 \%$, respectively (Fig. 1e).

\section{Case 2}

A 55-year-old female was referred to our hospital by a local physician due to elevated serum ALP, and a past medical history of Basedow disease was noted. CT imaging showed swelling of the skull, while ${ }^{99 \mathrm{~m}} \mathrm{Tc}-\mathrm{HMDP}$ bone SPECT/CT showed focal and intense uptake in the bony lesion (Fig. 2). Serum TRACP-5B and ALP before bisphosphonate treatment were $1,583 \mathrm{mU} / \mathrm{dL}$ and $762 \mathrm{U} / \mathrm{L}$, respectively. The patient was diagnosed with PDB in the skull, and risedronate sodium hydrate (17.5 mg, once daily) was prescribed. After 1 year of treatment, TRACP-5B and ALP were decreased to $181 \mathrm{mU} / \mathrm{dL}$ (88.6\% reduction) and $167 \mathrm{U} / \mathrm{L}$ (78.1\% reduction), respectively. ${ }^{99 \mathrm{~m}} \mathrm{Tc}-\mathrm{HMDP}$ bone SPECT/CT revealed decreased uptake in the bony lesion (Fig. 2). The $S_{U V} V_{\text {max }}, S V_{\text {peak }}, S_{\text {mean }}, \mathrm{MBV}$, and TBU for the mass before risedronate sodium hydrate treatment were $7.48,6.51,4.00,307.2 \mathrm{~mL}$, and 1,229.1 g, respectively, while those after treatment were decreased slightly to $2.97,2.68,1.58,342.4 \mathrm{~mL}$, and $543.8 \mathrm{~g}$, respectively, for reductions of $60.3,58.8,60.5,11.8$, and $55.8 \%$, respectively (Fig. 2).

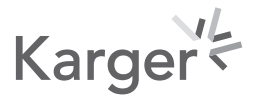


Kitajima et al.: Quantitative SPECT/CT for Response to Bisphosphonate in PDB

\section{Discussion}

PDB is a focal skeletal disorder characterized by formation of structurally abnormal bone and skeletal deformities, as well as other complications that lead to bone pain and significant disability. One or more areas in a single bone (monostotic) or multiple bones (polyostotic) can be involved, with the skull, pelvis, sacrum, lumbar spine, femur, and tibia the most common sites affected. In most cases, PDB is asymptomatic, and the diagnosis initially suspected incidentally based on increased levels of bone metabolism markers, especially ALP, and then confirmed by specific findings in radiography and bone scintigraphy. For evaluation of treatment response to bisphosphonate therapy, ALP and bone scintigraphy are generally used. Although it is technically difficult to quantify local tracer uptake using conventional bone scintigraphy, quantitative bone SPECT/CT has recently emerged as an accurate and noninvasive method that may be helpful for clinicians to decide whether bisphosphonate treatment should be prolonged or abandoned in affected patients.

State-of-the-art SPECT/CT produces objective quantitative data. Using results of robust algorithms of CT-based attenuation correction, scatter correction, and resolution recovery, SPECT/ CT generates imaging voxels denoted as units of radioactivity per volume (i.e., $\mathrm{kBq} / \mathrm{mL}$ ). This is fundamentally different from traditional nuclear imaging methods, such as planar scintigraphy, SPECT, and nonquantitative SPECT/CT, which use counts per second for their imaging units. With quantitative SPECT/CT, lesion radioactivity can be normalized for injected radioactivity, resulting in quantitative parameter values, such as percent injected dose and SUV [2-4]. Zeintl et al. [2] reported that advanced SPECT/CT technology facilitated quantitative ${ }^{99 \mathrm{~m}}$ Tc SPECT imaging with excellentaccuracy in both phantom (error $<3.6 \%$ ) and patient (error $<1.1 \%$ ) studies. Furthermore, Gnesin et al. [4] found in a phantom study that both absolute activity and the concentration of activity determined with quantitative ${ }^{99 \mathrm{~m}} \mathrm{Tc}$ SPECT/CT were within $10 \%$ of expected values.

Although several recently published studies have demonstrated the clinical application of quantitative SPECT/CT for evaluating treatment response in patients with bone disease [5-7], there are no known reports of quantitative SPECT/CT used for evaluating treatment response to bisphosphonates given to PDB patients. Beck et al. [5] investigated changes in SUV before and after treatment in 16 breast cancer and 3 prostate cancer patients (hormonal therapy and bisphosphonates) using skeletal quantitative SPECT/CT with ${ }^{99 \mathrm{~m}} \mathrm{Tc}$-diphosphonopropanedicarboxylic acid. They reported mean $S_{U V}$ peak and $S U V_{\text {mean }}$ values for 52 bone metastasis sites prior to treatment of $20.4 \pm 20.8$ and $5.6 \pm 1.9$, which were decreased following treatment to $16.4 \pm 11.5$ (19.6\% reduction) and $4.9 \pm 2.2$ (12.5\% reduction), respectively. Umeda et al. [6] evaluated the usefulness of three-dimensional TBU for evaluating active bone metastatic burden in 9 prostate cancer patients who underwent Ra-223 therapy and found that the change in TBU derived from the established threshold value (SUV >7.0) was more accurate and sensitive than the change in classic bone scan index in those cases. Furthermore, Kitajima et al. [7] reported a case of periosteal osteosarcoma in which $\operatorname{SUV}_{\text {max }}, \mathrm{SUV}_{\text {peak }}$, $\mathrm{SUV}_{\text {mean }}, \mathrm{MBV}$, and TBU values shown by quantitative bone SPECT/CT were useful to evaluate treatment response to preoperative chemotherapy.

\section{Conclusions}

Important indexes, including $\mathrm{SUV}_{\max }, \mathrm{SUV}_{\text {peak }}, \mathrm{SUV}_{\text {mean }}, \mathrm{MBV}$, and $\mathrm{TBU}$, determined using quantitative bone SPECT/CT findings, are useful to evaluate the activity of bone disease such as PDB as well as treatment response. We have found that quantitative bone SPECT/CT is noninvasive and useful for accurate monitoring of the efficacy of treatment response in affected patients. 


\section{Case Reports in Oncology}

Case Rep Oncol 2020;13:829-834

\begin{tabular}{l|c|}
\hline DOI: 10.1159/000507663 & ( 2020 The Author(s). Published by S. Karger AG, Basel
\end{tabular} www.karger.com/cro

Kitajima et al.: Quantitative SPECT/CT for Response to Bisphosphonate in PDB

\section{Statement of Ethics}

Written informed consent for publication of the case was obtained.

\section{Conflict of Interest Statement}

The authors have no conflicts of interest to declare.

\section{Funding Sources}

This work was supported by JSPS KAKENHI (grant No. 19K08187).

\section{Author Contributions}

Concept and design: K.K. and H.F.; acquisition of data: H.F., T.T., and Y.T.; drafting of the manuscript: K.K.; critical revision of the manuscript for important intellectual content: H.F., T.T., Y.T., and K.Y. All authors approved the final version of the manuscript.

\section{References}

1 Ralston SH, Corral-Gudino L, Cooper C, Francis RM, Fraser WD, Gennari L, et al. Diagnosis and management of Paget's disease of bone in adults: a clinical guideline. J Bone Miner Res. 2019;34(4):579-604.

2 Zeintl J, Vija AH, Yahil A, Hornegger J, Kuwert T. Quantitative accuracy of clinical 99mTc SPECT/CT using ordered-subset expectation maximization with 3-dimensional resolution recovery, attenuation, and scatter correction. J Nucl Med. 2010;51(6):921-8.

3 Bailey DL, Willowson KP. An evidence-based review of quantitative SPECT imaging and potential clinical applications. J Nucl Med. 2013;54(1):83-9.

4 Gnesin S, Leite Ferreira P, Malterre J, Laub P, Prior JO, Verdun FR. Phantom validation of Tc-99m absolute quantification in a SPECT/CT commercial device. Comput Math Methods Med. 2016;2016:4360371.

5 Beck M, Sanders JC, Ritt P, Reinfelder J, Kuwert T. Longitudinal analysis of bone metabolism using SPECT/CT and 99mTc-diphosphono-propanedicarboxylic acid: comparison of visual and quantitative analysis. EJNMMI Res. 2016;6(1):60.

6 Umeda T, Koizumi M, Fukai S, Miyaji N, Motegi K, Nakazawa S, et al. Evaluation of bone metastatic burden by bone SPECT/CT in metastatic prostate cancer patients: defining threshold value for total bone uptake and assessment in radium-223 treated patients. Ann Nucl Med. 2018;32(2):105-13.

7 Kitajima K, Futani H, Fujiwara M, Minakawa G, Osugi Y, Tsuchitani T, et al. Usefulness of quantitative bone single photon emission computed tomography/computed tomography for evaluating response to neoadjuvant chemotherapy in a patient with periosteal osteosarcoma. Cureus. 2018;10(11):e3655. 